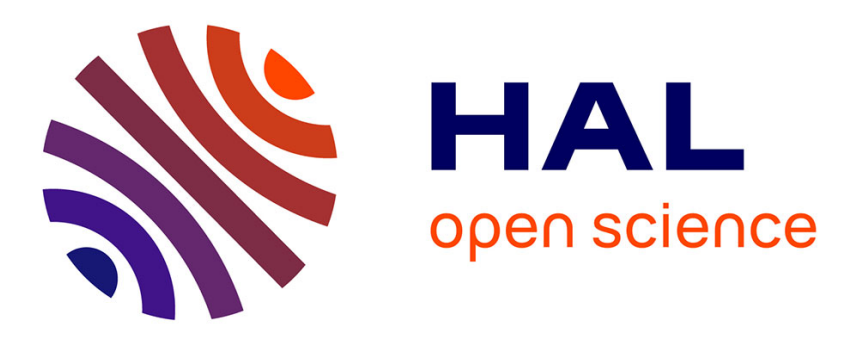

\title{
Ultrafast ultrasound coupled with cervical magnetic stimulation for non-invasive and non-volitional assessment of diaphragm contractility
}

Thomas Poulard, Martin Dres, Marie-Cécile Nierat, Isabelle Rivals, Jean-Yves Hogrel, Thomas Similowski, Jean-Luc Gennisson, Damien Bachasson

\section{To cite this version:}

Thomas Poulard, Martin Dres, Marie-Cécile Nierat, Isabelle Rivals, Jean-Yves Hogrel, et al.. Ultrafast ultrasound coupled with cervical magnetic stimulation for non-invasive and non-volitional assessment of diaphragm contractility. The Journal of Physiology, 2020, 598 (24), pp.5627-5638. 10.1113/JP280457 . hal-03266752

\section{HAL Id: hal-03266752 https://hal.sorbonne-universite.fr/hal-03266752}

Submitted on 22 Jun 2021

HAL is a multi-disciplinary open access archive for the deposit and dissemination of scientific research documents, whether they are published or not. The documents may come from teaching and research institutions in France or abroad, or from public or private research centers.
L'archive ouverte pluridisciplinaire HAL, est destinée au dépôt et à la diffusion de documents scientifiques de niveau recherche, publiés ou non, émanant des établissements d'enseignement et de recherche français ou étrangers, des laboratoires publics ou privés. 
The Journal of Physiology

https://jp.msubmit.net

\title{
JP-TFP-2020-280457R2
}

Title: Ultrafast ultrasound coupled with cervical magnetic stimulation for non-invasive and non-volitional assessment of diaphragm contractility

\author{
Authors: Thomas Poulard \\ Martin Dres \\ Marie-Cécile Nierat \\ Isabelle Rivals \\ Jean-Yves Hogrel \\ Thomas Similowski \\ Jean-Luc Gennisson \\ Damien Bachasson
}

Author Conflict: Martin Dres: MD received personal fees from Lungpacer. Jean-Luc Gennisson: JLG is a scientific consultant for Supersonic Imagine, Aix-en-Provence, France.

Author Contribution: Thomas Poulard: Conception or design of the work; Acquisition or analysis or interpretation of data for the work; Drafting the work or revising it critically for important intellectual content; Final approval of the version to be published; Agreement to be accountable for all aspects of the work Martin Dres: Conception or design of the work; Drafting the work or revising it critically for important intellectual content; Final approval of the version to be published; Agreement to be accountable for all aspects of the work Marie-Cécile Nierat: Conception or design of the work; Drafting the work or revising it critically for important intellectual content; Final approval of the version to be published; Agreement to be accountable for all aspects of the work 
Isabelle Rivals: Acquisition or analysis or interpretation of data for the work; Drafting the work or revising it critically for important intellectual content; Final approval of the version to be published; Agreement to be accountable for all aspects of the work JeanYves Hogrel: Conception or design of the work; Drafting the work or revising it critically for important intellectual content; Final approval of the version to be published; Agreement to be accountable for all aspects of the work Thomas Similowski: Conception or design of the work; Drafting the work or revising it critically for important intellectual content; Final approval of the version to be published; Agreement to be accountable for

all aspects of the work Jean-Luc Gennisson: Conception or design of the work;

Acquisition or analysis or interpretation of data for the work; Drafting the work or revising it critically for important intellectual content; Final approval of the version to be published; Agreement to be accountable for all aspects of the work Damien Bachasson: Conception or design of the work; Acquisition or analysis or interpretation of data for the work; Drafting the work or revising it critically for important intellectual content;

Final approval of the version to be published; Agreement to be accountable for all aspects of the work

Running Title: Ultrafast ultrasound imaging of the diaphragm

\section{Dual Publication: No}

Funding: Fondation EDF: Thomas Poulard, Jean-Yves Hogrel, Damien Bachasson, N/A; Association Francaise contre les Myopathies (Association Française contre les Myopathies): Thomas Poulard, Jean-Yves Hogrel, Damien Bachasson, N/A The PhD fellowship of TP is funded by the Fondation EDF that is supporting the RespiMyo project, which includes the current study. This study was also supported by the Association Française Contre Les Myopathies (AFM). 


\section{Ultrafast ultrasound coupled with cervical magnetic 2 stimulation for non-invasive and non-volitional 3 assessment of diaphragm contractility}

4 Thomas Poulard ${ }^{1,2}$, Martin Dres ${ }^{3,4}$, Marie-Cécile Niérat ${ }^{3}$, Isabelle Rivals ${ }^{5}$, Jean-Yves

5 Hogrel $^{2}$, Thomas Similowski ${ }^{3,4}$, Jean-Luc Gennisson ${ }^{1 \#}$, Damien Bachasson ${ }^{2 \#^{*}}$

6 \# equally contributing authors

$9{ }^{1}$ Laboratoire d'Imagerie Biomédicale Multimodale, BioMaps, Université Paris-Saclay, 10 CEA, CNRS UMR 9011, Inserm UMR1281, SHFJ, 4 place du général Leclerc, 91401, Orsay, 11 France

$12{ }^{2}$ Institute of Myology, Neuromuscular Investigation Center, Neuromuscular Physiology 13 Laboratory, Paris, France

$14{ }^{3}$ Sorbonne Université, INSERM, UMRS1158 Neurophysiologie respiratoire expérimentale 15 et clinique, Paris, France

$16{ }^{4}$ AP-HP. Sorbonne Université, Hôpital Pitié-Salpêtrière, Service de Pneumologie, Médecine 17 intensive - Réanimation (Département "R3S"), F-75013, Paris, France

$18{ }^{5}$ Equipe de Statistique Appliquée, ESPCI Paris, PSL Research University, UMRS 1158, 10

19 rue Vauquelin, 75005, Paris, France

21 *Corresponding author: Damien Bachasson, PhD. Institut de Myologie, Laboratoire de 22 Physiologie et d'Evaluation Neuromusculaire, Hôpital Universitaire Pitié Salpêtrière, Paris 2375651 Cedex 13, France. Tel: +33 1421666 41; fax: +33 1421658 81. E-mail: 24 d.bachasson@institut-myologie.org 


\section{Table of contents categories}

26 Respiratory

\section{Key points summary}

29 - Twitch transdiaphragmatic pressure elicited by cervical magnetic stimulation of the phrenic nerves is a fully non-volitional method for assessing diaphragm contractility in humans, yet it requires invasive procedures such as esophageal and gastric catheterballoons.

- Ultrafast ultrasound enables a very high frame rate allowing the capture of transient events, such as muscle contraction elicited by nerve stimulation (twitch). Whether indices derived from ultrafast ultrasound can be used as an alternative to the invasive measurement of twitch transdiaphragmatic pressure is unknown.

- Our findings demonstrate that maximal diaphragm tissue velocity assessed using ultrafast ultrasound following cervical magnetic stimulation is reliable, sensitive to change in cervical magnetic stimulation intensity, and correlates to twitch transdiaphragmatic pressure.

- This approach provides a novel fully non-invasive and non-volitional tool for the assessment of diaphragm contractility in humans. 


\section{Abstract}

44 Measuring twitch transdiaphragmatic pressure (Pditw) elicited by cervical magnetic 45 stimulation (CMS) is considered as a reference method for the standardized evaluation of 46 diaphragm function. Yet, the measurement of Pdi requires invasive esophageal and gastric 47 catheter-balloons. Ultrafast ultrasound is a non-invasive imaging technique enabling frame 48 rates high enough to capture transient events such as evoked muscle contractions. This study

49 investigated relationships between indices derived from ultrafast ultrasounds and Pditw, and 50 how these indices may be used to estimate Pditw. CMS was performed in 13 healthy 51 volunteers from 30 to $100 \%$ of stimulator intensity in units of $10 \%$ in a randomized order. 52 Pditw was measured and the right hemidiaphragm was imaged using a custom ultrafast 53 ultrasound sequence with $1 \mathrm{kHz}$ framerate. Maximal diaphragm axial velocity (Vdimax) and 54 diaphragm thickening fraction $\left(\mathrm{TFdi}_{\mathrm{tw}}\right)$ were computed. Intra-session reliability was 55 assessed. Repeated-measures correlation (R) and Spearman correlation coefficients $(\rho)$ were 56 used to assess relationships between variables. Intra-session reliability was strong for Pditw 57 and Vdimax and moderate for TFditw. Vdimax correlated with Pditw in all subjects $(0.64<\rho<$ 58 1.00, $\mathrm{R}=0.75$; all $p<0.05)$. TFditw correlated with Pditw in 8 subjects only $(0.85<\rho<0.93$, $59 \mathrm{R}=0.69$; all $p<0.05)$. Coupling ultrafast ultrasound and CMS show promise for the non60 invasive and fully non-volitional assessment of diaphragm contractility. This approach opens 61 up prospects for both diagnosis and follow-up of diaphragm contractility in clinical 62 populations.

63

64 Key Words: Diaphragm, ultrafast ultrasound imaging, cervical magnetic stimulation, 65 skeletal muscle, contractility, phrenic nerves 


\section{Introduction}

Sixty years ago, Agostoni \& Rahn, (1960) introduced a novel method to measure the specific contribution of the diaphragm to the intrathoracic pressure generated during inspiratory efforts, namely, transdiaphragmatic pressure (Pdi). Pdi is defined as the difference between gastric (Pga) and esophageal (Pes) pressures measured using gastric and esophageal probes. Twitch Pdi (Pditw) elicited by cervical magnetic stimulation (CMS) was introduced 30 years ago and is considered as a reference method for the non-volitional assessment of diaphragm contractility (Similowski et al., 1989). Yet, measuring Pditw is considered invasive and requires a high level of expertise (Laveneziana et al., 2019). Twitch mouth pressure (Pmotw) or nasal mask twitch pressure have been developed as an alternative to Pditw (Yan et al., 1992; Teixeira et al., 2007). However, this approach requires some degree of cooperation

77 from the subjects because small inspiratory/expiratory efforts (Similowski et al., 1993; Hamnegaard et al., 1995; Windisch et al., 2005; Kabitz et al., 2007) are required prior the stimulation to prevent upper airway collapse and/or glottis closure and ensure adequate transmission. Moreover, these procedures required proper mouth occlusion, which cannot be performed in many patients such as patients with neuromuscular disorders.

Ultrasound (US) imaging has emerged as a tool for assessing the diaphragm (Ueki et

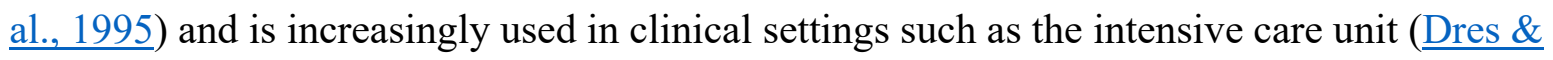
Demoule, 2020). Imaging of the zone of apposition of the right-hemidiaphragm is classically performed to investigate diaphragm behavior. Various indices can be derived from diaphragm US such as diaphragm excursion or thickening fraction (Goligher et al., 2015;

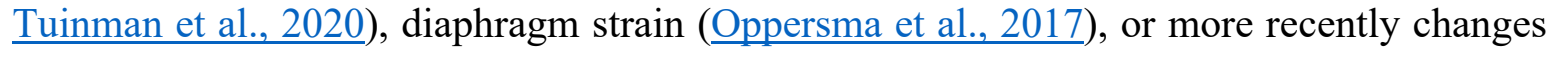
in diaphragm stiffness assessed with US shear wave elastography (Bachasson et al., 2019). However, these methods offer limited frame rate (i.e. a few tens of frames per second for

91 methods cannot be used for capturing fast transient phenomena, such as diaphragm response 92 elicited by CMS ( $300 \mathrm{~ms})$.

93 Ultrafast US is a fairly recent imaging technique enabling very high frame rates (up 94 to $20 \mathrm{kHz}$, (Sandrin et al., 1999). This technique has previously been used in the biceps 
95 brachii to visualize muscle behavior during short-lasting contractions (Deffieux et al., 2008;

96 Gronlund et al., 2013). By performing a radio frequency-based speckle tracking, ultrafast US

97 allows the quantification of transient velocities of mechanical waves induced by 98 transcutaneous electrical stimulation (Deffieux et al., 2008). Maximal tissue velocity has

99 been reported to increase linearly with stimulation intensity. However, the relationship

100 between tissue velocity and the force generated by the muscle during stimulation is unknown.

101 In a recent pilot work, we reported that diaphragm response elicited by CMS can be imaged

102 using ultrafast US and that responses elicited at high and low stimulation intensity can be

103 discriminated (Bachasson et al., 2018). However, the relationship between diaphragm

104 pressure generation and indices derived from ultrafast US during CMS remains to be 105 thoroughly investigated.

106 Therefore, this study aimed at imaging the diaphragm during CMS at different 107 intensity levels using ultrafast US. By investigating the relationships between Pditw and 108 indices derived from ultrafast US imaging (i.e. thickening fraction, maximal tissue velocity), 109 we hypothesized that diaphragm thickening fraction and diaphragm tissue velocity following 110 CMS were correlated to Pditw, and that these indices may be used as a surrogate to Pditw.

\section{Methods}

\section{Ethical approval}

113 This study conformed to the Declaration of Helsinki. It was approved by the local 114 ethics committee (Comité de Protection des Personnes Île-de-France VI, France, February $11522^{\text {nd }}$ 2016, ID-RCB 2015-A00949-40) and was publicly registered before the first inclusion 116 (ClinicalTrials.gov, NCT03313141). All participants gave written informed consent. Some 117 of the data from this study have already been published elsewhere, regarding the use of 118 diaphragm shear wave elastography in healthy subjects during ventilation (Bachasson et al., 119 2019).

\section{Participants}


Thirteen healthy participants (5 males and 8 females, median (Q1-Q3) - age $=24$ (22-

122 27) years, height $\left.=171(167-183) \mathrm{cm}, \mathrm{BMI}=20.6(19.7-22.6) \mathrm{kg} \cdot \mathrm{m}^{-2}\right)$ were studied.

123 Participants had to be 18 and over with no history of respiratory or neuromuscular disorders,

124 and no contraindication to CMS (Rossi et al., 2011).

\section{Pressure measurements}

126 Participants were studied in a semirecumbent position ( 45 degrees) with uncast 127 abdomen. Pes and Pga were measured using $8 \mathrm{~cm}$ balloon catheters (Marquat Genie 128 Biomedical, Boissy-Saint-Léger Cedex, France). Balloons were introduced through the 129 participant's nostril and both placed in the stomach so that a positive pressure deflection was 130 monitored when gently pressing the participant's stomach. Subsequently, one balloon was 131 slowly withdrawn toward the esophagus until the pressure deflection was no more monitored 132 when pressing the participant's stomach, and was then withdrawn an additional $10 \mathrm{~cm}$. 133 Esophageal balloon position was adjusted using the Baydur maneuver (Baydur et al., 1982). 134 Balloons were then connected to differential pressure transducers (MLT0380/D, 135 ADInstruments, Bella Vista, Australia) and filled with 4 and $5.5 \mathrm{ml}$ of air in the esophageal 136 and gastric balloons, respectively (Mojoli et al., 2015). All signals were digitized at a $4 \mathrm{kHz}$ 137 frequency using a PowerLab system (16/35, ADInstruments, Bella Vista, Australia) and 138 recorded on the LabChart software. Pdi was computed as the difference between Pga and 139 Pes.

\section{Cervical Magnetic Stimulation}

141 CMS was performed using a Magstim 200 stimulator (Magstim, Whitland, Dyfed, 142 UK) driving a 90-mm circular coil (1 Tesla maximum output) as previously described 143 (Similowski et al., 1989). Briefly, participants were asked to bend their neck forward and the 144 central hole of the coil was positioned on the spinous process of the seventh cervical vertebra. 145 Optimal coil position was determined by performing a series of stimulation at $100 \%$ of 146 stimulator intensity. The spot where Pditw was the highest was skin-marked and kept constant 147 during the whole experiment.

148 Ultrafast ultrasound imaging 
The zone of apposition of the right hemidiaphragm was imaged using a $6 \mathrm{MHz}$ central 150 frequency linear transducer (SL 10-2) driven by an ultrafast ultrasound device (Aixplorer 151 V12, Supersonic Imagine, Aix-en-Provence, France). The probe was placed on the mid152 axillary line, vertical to the chest wall, at the 8th-10th intercostal space. The site of the probe 153 placement was skin-marked to ensure that the same region of interest was imaged during the 154 whole protocol. The diaphragm was identified as a three-layers structure superficial to the 155 liver, with two hyperechoic layers (i.e. the pleura and peritoneum) surrounding a hypoechoic 156 muscular layer (Figure 1). As the duration of Pditw is $\sim 300 \mathrm{~ms}$, a custom ultrafast US 157 sequence was designed to track diaphragm movements during this time window. The 158 sequence was composed of 9 plane-wave US with different angles $\left(-7^{\circ}\right.$ to $7^{\circ}$ with a $2^{\circ}$ 159 incremental steps) at $9 \mathrm{kHz}$ frame rate, yielding a compounded frame rate of $1 \mathrm{kHz}$ and a 500 $160 \mathrm{~ms}$ (Montaldo et al., 2009). This sequence followed the Food and Drugs Administration 161 guidelines for acoustics norms (Mechanical index $=0.5$, Thermal index $=0.2$ ). Because 162 diaphragm depth rarely exceed $4 \mathrm{~cm}$ (Shahgholi et al., 2014), the US sequence was developed 163 in order to maintain the same spatial and temporal resolution of to this depth of $4 \mathrm{~cm}$. Such 164 sequence allows the imaging of the diaphragm in overweight patients. Signals were 165 synchronized using an output trigger sent from the ultrafast US device to the Powerlab 166 system. A fixed delay of $100 \mathrm{~ms}$ was set between the onset of US recordings and CMS, after 167 which the stimulator was triggered by the Powerlab for delivering the stimulation. Recording 168 of pressure signals was started $1 \mathrm{~s}$ before the US trigger. The experimental setup and 169 procedure for recording pressure and US frames is displayed in Figure 2. Of note, we 170 investigated whether diaphragm excursion elicited by CMS may be imaged during subcostal 171 scanning during pilot works. We measured very small excursion values that were highly 172 variable between trials. This finding was expected as diaphragm response elicited by CMS 173 is not associated with substantial change in pulmonary volume. This may be mainly

174 explained by glottis closure. Consequently, the measurement of diaphragm excursion during 175 CMS was not further explored.

\section{Experimental protocol}


177 Cervical magnetic stimulations. Participants were stimulated on the predefined optimal 178 stimulation spot from 30 to $100 \%$ of stimulator intensity in units of $10 \%$, in a randomized 179 order. All stimulations were delivered at functional residual capacity (FRC). Lung volume 180 prior stimulation, estimated through Pes, was checked to be consistent across all stimulations. 181 A minimum of three stimulations, separated by at least one minute, were performed at each 182 stimulation intensity. Two to three validated trials (i.e. as indicated by appropriate Pes before 183 CMS) per intensity were considered for further analysis.

184 Maximal voluntary maneuvers. Participants were asked to perform maximal inspiratory 185 effort at residual volume. Maximal Pdi (Pdimax) was measured using a unidirectional valve 186 allowing expiration only. Participants were asked to empty their lungs before being strongly 187 encouraged to generate maximal inspiratory effort. Visual feedback of Pdi was provided 188 during the maneuver. Three to five trials were performed and maximal pressure measured 189 over a $1 \mathrm{~s}$ period was recorded as Pdimax. Sniff nasal inspiratory pressure (SNIP) was 190 determined as follows. Participants were asked to make a short and maximal sniff at FRC. 191 As recommended (American Thoracic Society/European Respiratory, 2002), participants 192 performed 8-10 attempts with a $\sim 30$-s rest in-between sniffs until a plateau of peak pressure 193 values was reached.

\section{Data analysis}

195 All data were analyzed offline using standardized Matlab scripts (Mathworks, Natick,

196 MA, USA). Pes, Pga, and Pdi signals were low-pass filtered (30 Hz) using a second-order 197 Butterworth filter. Esophageal twitch pressure (Pestw), gastric twitch pressure (Pgatw), and 198 Pdi $_{\text {tw }}$ following stimulation were calculated as the difference between maximal (for Pdi and 199 Pga) or minimal (for Pes) pressure and pressure at the onset of CMS.

200 Vertical speckle tracking was performed by computing the axial (i.e. perpendicular 201 to the ultrasound probe) relative displacements within the diaphragm. This technique consists 202 in comparing consecutive images using one-dimensional cross-correlations to measure the 203 relative displacement of a pixel between two consecutive frames (Loupas et al., 1995). 204 Diaphragm tissue velocity profile is then computed by dividing the measured displacement 205 by the time difference between two frames (i.e. $1 \mathrm{~ms}$ ). As an example, Figure 3 shows how 
206 the velocity within the diaphragm evolves over time. Diaphragm velocity was computed over 207 each column of pixels within the diaphragm. The central third of each image was then 208 averaged to obtain a single value of diaphragm velocity over time. This value was assumed 209 to be representative of the whole diaphragm. Maximal diaphragm velocity (Vdimax) was then 210 determined as the maximal (i.e. positive) velocity within this signal.

211 For each trial, a time-motion image was generated using the central pixel line of each 212 ultrasound image, referred to as M-Mode in the following. The position of the pleura and 213 peritoneum layers was then drawn manually over the full length of the M-Mode image. By 214 doing so, diaphragm thickness (i.e. the difference between the peritoneum and pleura 215 positions) was computed at each time of the US acquisition. Maximal diaphragm thickening 216 fraction (TFditw) was computed using resting diaphragm thickness prior stimulation (Tdi ${ }_{\text {rest }}$ ) 217 and maximal diaphragm thickness following stimulation (Tdimax $)$ as follows:

$$
\operatorname{TFdi} \mathrm{iw}_{\mathrm{tw}}(\%)=\frac{\mathrm{Tdi}_{\mathrm{max}}-\mathrm{Tdi}_{\text {rest }}}{\mathrm{Tdi}_{\text {rest }}} \times 100
$$

219 All TFditw measurements were performed by a single trained operator (TP), blinded to the 220 stimulation intensity. A movie clip showing pressure signals, M-mode images, and indices 221 derived from ultrafast US is available in supplementary materials S1.

\section{Statistics}

223 Results are presented as median (Q1-Q3) unless otherwise stated. Normality was assessed by 224 visual inspection (QQ plots and density distributions) and by significance tests (Shapiro-Wilk 225 test). Because all variables failed the normality test, Friedman repeated measures ANOVAs 226 were used. ANOVAs were conducted to compare Pes prior to each stimulation at all 227 stimulation intensities. Within-day reliability of Pditw, Vdimax, and TFditw was investigated.

228 Standard errors of measurement (SEM) and intraclass correlation coefficients (ICC) were 229 used to study absolute and relative reliability, respectively (Hopkins, 2002). The overall 230 relationship between variables $(\mathrm{R})$ was determined using repeated measure correlation 231 (Bakdash \& Marusich, 2017). This technique considers the independence of repeated 232 measures between individuals, so that potential confounding factors, such as between233 participant variability, do not interfere. Data are presented as R [95\% CI]. Spearman 
234 correlation coefficients $(\rho)$ were calculated to investigate within-individual relationships 235 between variables. ANOVAs were used to assess the effect of stimulation intensity on Pditw,

236 Vdimax, and TFditw. Tukey's post-hoc tests were conducted if a significant main effect of 237 intensity was found. Within individuals, Pditw, Vdimax, and TFdi tw $_{\text {were }}$ considered 238 supramaximal if the average $\mathrm{Pdi}_{\mathrm{tw}}, \mathrm{Vdi}_{\max }$ or $\mathrm{TFdi} \mathrm{i}_{\mathrm{tw}}$ at submaximal and maximal stimulation 239 intensities was inferior or equal to the coefficient of variation of the variable at each 240 stimulation intensities (Welch et al., 2018; Geary et al., 2019). Supramaximality was reached 241 if greater stimulation intensity did not result in further increase in Pditw, Vdimax or TFditw. 242 Analyses were performed in the computing environment $\mathrm{R}$ ( $\mathrm{R}$ Core Team, 2020). 243 Significance was set at $p<0.05$ for all tests.

\section{Results}

All participants completed the protocol. Pdimax was 113 (71-115) $\mathrm{cmH}_{2} \mathrm{O} 90$ (56-117) $\mathrm{cmH}_{2} \mathrm{O}$ in men and women, respectively. Overall, Pdimax was 108 (71-117) $\mathrm{cmH}_{2} \mathrm{O}$. SNIP was $116(109-130) \mathrm{cmH}_{2} \mathrm{O}$ in men and $103(90-118) \mathrm{cmH}_{2} \mathrm{O}$ in women. Overall, SNIP was $109(96-123) \mathrm{cmH}_{2} \mathrm{O}$. The one-way repeated measures ANOVA showed that Pes at the onset of CMS was similar across all stimulations at all intensities $(p=0.2430)$. Within-day SEM and ICC of Pditw, Vdi max, and TFditw are presented in Table 1. Typical B-Mode images over the course of the $500 \mathrm{~ms}$ US acquisition are presented in supplementary materials S2.

\section{Effect of stimulation intensity on indices derived from ultrafast ultrasound}

M-Mode images and temporal evolution of the displacements of the pleura and peritoneum, $\mathrm{Vdi}_{\max }$, and recorded pressures in one individual are displayed in Figure 4 (also see movie clip in Supporting Information S1). Pestw, Pgatw, and Pditw at all tested stimulation intensities are shown in Figure 5 and Figure 6A. Vdimax and TFditw at all tested stimulation

257 intensities are displayed in Figure 6B-C. Within individual relationships between stimulation 258 intensity and Pditw, Vdimax, and TFditw are shown in Figure 6D-F.

Pditw was significantly related to stimulation intensity in all subjects ( $\rho$ ranged from 2600.83 to 1.00 , all $p<0.0100 ; \mathrm{R}=0.91,95 \%$ CIs [0.86 0.94], $p<0.0001)$. At the group level, 261 there was a significant main effect of stimulation intensity on Pditw. Post-hoc tests indicated 
262 that Pditw significantly increased up to $100 \%$ of stimulation intensity (all $p<0.05$ ). Within 263 individuals, Pditw plateaued at $90 \%$ of stimulation intensity in two participants. In other 264 participants, Pditw increased until $100 \%$ of stimulation intensity.

265 Vdimax correlated to stimulation intensity in all participants ( $\rho$ ranged from 0.79 to 2661.00 , all $p<0.0500 ; \mathrm{R}=0.83,95 \%$ CIs [0.75 0.89], $p<0.0001)$. At the group level, there was 267 a significant main effect of stimulation intensity on Vdimax. Post-hoc tests indicated that 268 Vdimax did not significantly differ between 90 and $100 \%$ of stimulation intensity $(p=0.9997)$.

269 No significant differences in Vdimax was found between consecutive stimulation intensities, 270 except between 80 and $90 \%$ of stimulation intensity $(p=0.0080)$. Within individuals, Vdimax 271 plateaued at $90 \%$ of stimulation intensity in 6 participants, at $80 \%$ in one participant, and at $27270 \%$ in one participant.

TFditw correlated to stimulation intensity $(\mathrm{R}=0.72,95 \% \mathrm{CIs}[0.600 .80], p<0.0001)$.

274 Individual correlations were significant in 10 out of 13 subjects ( $\rho$ ranged from 0.67 to 0.95 , 275 all $p<0.05 ; \rho$ ranged from 0.33 to 0.52 in the three remaining participants, all $p>0.2200$ ). At 276 the group level, there was a significant main effect of stimulation intensity on TFditw. Post$277 h o c$ tests showed that TFditw did not significantly differ between 60 to $100 \%$ of stimulation 278 intensity (all $p>0.1155$ ). No significant differences in TFditw was found between consecutive 279 stimulation intensities. Within individuals, TFditw plateaued at $90 \%$ of stimulation intensity 280 in 5 participants, at $80 \%$ in two participants, at $70 \%$ in two participants, at $50 \%$ in one 281 participant and at $30 \%$ in one participant. TFditw and $\mathrm{Vdi}_{\max }$ at all stimulation intensities are 282 shown in Figure 6B and 6C, respectively.

\section{Relationships between Pditw and indices derived from ultrafast ultrasound}

Within-individuals' relationships between Pditw and Vdimax, and between Pditw and TFditw are presented in Figure 7. Vdimax correlated to Pditw in all participants ( $\rho$ ranged from 0.64 to 1.00 , all $p<0.05 ; \mathrm{R}=0.75,95 \%$ CIs [0.65 0.83], $p<0.0001$ ). TFditw positively 287 correlated to $\mathrm{Pditw}_{\mathrm{tw}}(\mathrm{R}=0.69,95 \% \mathrm{CIs}$ [0.57 0.79], $p<0.0001)$ and individual correlation coefficients were significant in 8 out of 13 participants ( $\rho$ ranged from 0.85 to 0.93 , all $p<0.05 ; \rho$ ranged from -0.27 to 0.70 , in the five remaining participants, all $p>0.06$ ). 


\section{Discussion}

291 This study is the first to image the diaphragm contraction induced by CMS using 292 ultrafast US. The main results are as follow: i) maximal tissue velocity within the diaphragm

293 significantly increased with stimulation intensity while diaphragm thickening fraction

294 plateaued at low stimulation intensity, ii) intra-session reliability of maximal tissue velocity

295 within the diaphragm was high and intra-session reliability of diaphragm thickening fraction

296 was poor iii) twitch transdiaphragmatic pressure strongly correlated with maximal tissue

297 velocity within the diaphragm and moderately correlated with diaphragm thickening fraction.

298 Vdi $_{\text {max }}$ is sensitive to changes in stimulation intensity and correlates to twitch 299 transdiaphragmatic pressure

300 We found that Vdimax increased with stimulation intensity in all participants. These 301 results are in line with previous works that reported a gradual increase in tissue velocity with 302 stimulation intensity during contractions elicited in the biceps brachii (Deffieux et al., 2008; 303 Gronlund et al., 2013). To the best of our knowledge, this study is the first to report the 304 relationship between a muscle's tissue velocity and the force/pressure it produces. We found 305 that Vdimax correlated to Pditw in all participants, supporting that the magnitude of Vdimax is 306 associated with the diaphragm contractility. Interestingly, Pditw was supramaximal in two 307 subjects only, whereas $\mathrm{Vdi}_{\max }$ was supramaximal in 8 subjects. The inability to reach 308 supramaximal Pditw values in some subjects has been addressed before (Man et al., 2004; 309 Spiesshoefer et al., 2019). This may be partly explained by insufficient magnetic stimulation 310 power to fully activate the phrenic nerves. It cannot be ruled out that supramaximality of 311 Pditw occurred between 90 and $100 \%$ of stimulation intensity. Nonetheless, it is known that 312 CMS at highest stimulation intensities stimulates neck muscles (Attali et al., 1997). Thus, 313 Pditw is likely to increase not because of a higher activation of the diaphragm, but because 314 the recruitment of neck muscles increases the deflation of twitch Pes (Wragg et al., 1994; 315 Laghi et al., 1996). Regarding Vdimax, supramaximality was reached in 8 out of 13 316 participants. The fact that Vdimax plateaued while Pditw continued to increase may be related 317 to specificity of Vdimax measurement, which directly probe the diaphragm. Therefore, Vdimax 318 may be considered as a specific index of diaphragm contractility following CMS, ruling out 
319 the confounding effects related to the recruitment of extra diaphragmatic muscle. Vdimax did 320 not meet supramaximality criteria in 5 subjects. Interestingly, 4 out of these 5 subjects did 321 not reach supramaximality for Pditw either. It can thus be suggested that the absence of Vdimax 322 supramaximality is directly related to the absence of Pditw supramaximality. Also, one may 323 observe that Vdi increases in the milliseconds following stimulation, while Pdi peaks $\sim 150$ $324 \mathrm{~ms}$ after stimulation (Figure 4). This supports that the rapid change in Vdi correspond to 325 diaphragm contraction and that this lag reflects the time needed for the diaphragm to transfer 326 its force generation into an actual pressure generation. Importantly, Vdimax was found to be 327 strongly reproducible, as indicated by low SEM and high ICC that were comparable to those 328 observed for Pditw (Table 1). This high reliability build confidence regarding the potential of 329 Vdimax for non-volitional monitoring of diaphragm contractility over time. This study is also 330 the first to report TFditw values during CMS. Repeated measure correlations showed a 331 significant correlation between TFditw and stimulation intensity. Within individuals, 10 (77 $332 \%$ p participants presented with a significant relationship between TFditw and stimulation 333 intensity. As compared to $\mathrm{Vdi}_{\max }$, TFditw was shown to be less sensitive to changes in 334 stimulation intensity. At the group level, TFditw was moderately correlated to Pditw. Our 335 results are in line with previous studies that reported significant relationship between 336 diaphragm thickening fraction and changes in Pdi during spontaneous breathing, inspiratory 337 efforts, or in mechanically ventilated patients (Ueki et al., 1995; Vivier et al., 2012; Goligher 338 et al., 2015; Umbrello et al., 2015). However, when looking at individual relationship, the correlation between TFditw and $\mathrm{Pdi}_{\mathrm{tw}}$ reached significance in 8 subjects $(62 \%)$ only. We also found that $\mathrm{TFdi}_{\mathrm{tw}}$ plateaued at low stimulation intensity (60\%). Importantly, the intra-session

341 reliability of $\mathrm{TFdi}_{\mathrm{tw}}$ was rather poor. There are several potential explanations for these 342 findings. First, TFditw was computed manually by drawing the position of the pleura and 343 peritoneum. Imprecisions during this manual step might also be amplified by the lower image 344 quality found using ultrafast US as compared to that of conventional US imaging. More 345 specifically, conventional US imaging uses focused pulses, allowing for high image quality 346 but a relatively low sampling rate (a few tens per second). On the other hand, ultrafast US 347 plane wave imaging allows very high sampling rate (here, $1 \mathrm{kHz}$ ) but image quality is lower 348 (Montaldo et al., 2009). Indeed, ultrafast US prevents the focusing of US beams to a specific 
349 tissue (i.e. in this case, the diaphragm) and negatively impacts the signal to noise ratio of the 350 resulting image. As a result, the ultrafast US sequence developed for the current experiment

351 does not allow strong contrast of anatomic structures in comparison to standard US imaging

352 (Figure 1A). This may disrupt the measurement of TFditw (Figure 1B) and contribute to 353 explain the low intra-session reliability of TFditw indicated by the substantial SEM $(\sim 10 \%)$ 354 and moderate ICC $(<0.6)$. Noteworthy, TFdi during ventilation was previously shown to be 355 moderately reliable using traditional ultrasound imaging (Goligher et al., 2015). This low 356 reliability may explain, at least in part, the absence of increase in TFditw with increasing 357 stimulation intensity and increasing Pditw in some participants. Indeed, we found in some 358 participants that TFditw plateaued at intensities as low as $40-60 \%$. Because of the large 359 increase in Pditw between 60 and $100 \%$ of stimulation intensity, it is very unlikely that 360 supramaximal TFditw values depicts full diaphragm recruitment. All together, these findings 361 suggest that TFditw may be of limited help to assess diaphragm contractility in response to 362 CMS.

363 Perspectives and limitations

364 We demonstrated that Vdimax was strongly related to Pditw in all subjects. This could 365 have important implications for monitoring temporal changes in diaphragm contractility in 366 patients presenting with diaphragm dysfunction. In other words, Vdimax elicited by CMS 367 could be monitored over time using ultrafast US, allowing iterative, specific, fully non368 invasive and non-volitional assessment of diaphragm contractility. It is worth noting that 369 between-subject variability was relatively important. In turn, one may question how Vdimax 370 may be used to identify diaphragm dysfunction. Further studies will focus on this specific 371 point, with the perspective that $\mathrm{Vdi}_{\max }$ may be one parameter, among others, guiding 372 clinicians through the assessment of diaphragm contractility. Inter-operator and between day 373 reliability of $\mathrm{Vdi}_{\max }$ remains to be investigated. Assessing the delay between CMS and 374 diaphragm response as assessed using Vdimax may also be promising to investigate both 375 phrenic conduction and electromechanical delay. Unfortunately, EMG was not available in 376 the current study and this shall be investigated in future works. As mentioned above, we 377 cannot ensure that supramaximality was achieved in all subjects. It is possible that 
378 maximality occurred between 90 and $100 \%$ of stimulation intensity in some subjects. This

379 problem has been addressed before (Man et al., 2004; Spiesshoefer et al., 2019), but can be

380 considered negligible if a rigorous and standardized study-design is routinely used. Also, as

381 the primary aim of this study was to detect changes in diaphragm contractility according to

382 stimulation intensity and relationships between variables, supramaximality should not be

383 considered as an important concern. We also emphasize that only the right hemidiaphragm

384 was imaged in this study and that future works shall thoroughly investigate this approach in

385 the left hemidiaphragm. Lastly, the ultrafast US sequence used in this study was custom-

386 made so that the present approach cannot be readily generalized to clinical environments as

387 it required a specific US scanner, US sequences that are not available commercially, specific

388 training, and represent a non-negligible costs.

389 Conclusion

390 These study shows that ultrafast US may be used to image diaphragm behavior

391 following CMS. Diaphragm tissue velocity is strongly correlated with twitch

392 transdiaphragmatic pressure and appears to be highly specific to diaphragm contractility.

393 Further research is warranted to investigate how ultrafast US may be used in patients, in

394 particular those with diaphragm dysfunction. Coupling ultrafast US with CMS opens

395 prospect for a fully non-invasive, non-volitional assessment and follow-up of diaphragm

396 contractility in clinical populations. 


\section{Additional information}

398 Data Availability Statement

The data that support the findings of this study are available from the corresponding

400 author upon reasonable request.

\section{Competing interests}

402 JLG is a scientific consultant for Supersonic Imagine, Aix-en-Provence, France. MD 403 received personal fees from Lungpacer.

404 Fundings

The PhD fellowship of TP is funded by the Fondation EDF that is supporting the 406 RespiMyo project, which includes the current study. This study was also supported by the 407 Association Française Contre Les Myopathies (AFM).

408 Author contributions

All authors participated in the conception and design of the study. TP and DB 410 performed experiments. TP, JLG and DB analyzed the data and drafted the original version 411 of the manuscript. All authors critically revised and approved the final version of the 412 manuscript. All persons designated as authors qualify for authorship, and all those who 413 qualify for authorship are listed.

\section{Supporting information}

415 S1. A movie clip, slowed down 40 times, showing pressure signals, M-mode images, 416 and indices derived from ultrafast US is available at the following link: 417 https://figshare.com/s/fe55c9aa033cb6a42617.

418 S2. A movie clip, in real-time, showing B-Mode and M-Mode images during a typical 419 500-ms ultrafast ultrasound acquisition is available at the following link: 420 https://figshare.com/s/79fb82dd0361075df33e 


\section{$421 \quad$ References}

422

423 Agostoni E \& Rahn H. (1960). Abdominal and thoracic pressures at different lung volumes. J Appl Physiol 15, 1087-1092.

425

American Thoracic Society/European Respiratory S. (2002). ATS/ERS Statement on respiratory muscle testing. Am J Respir Crit Care Med 166, 518-624.

428

Attali V, Mehiri S, Straus C, Salachas F, Arnulf I, Meininger V, Derenne JP \& Similowski T. (1997). Influence of neck muscles on mouth pressure response to cervical magnetic

432

Bachasson D, Dres M, Nierat M, Doorduin J, Gennisson J, Hogrel J \& Similowski T. (2018). Function. In 2018 IEEE International Ultrasonics Symposium (IUS), pp. 1-4.

Bachasson D, Dres M, Nierat MC, Gennisson JL, Hogrel JY, Doorduin J \& Similowski T. (2019). Diaphragm shear modulus reflects transdiaphragmatic pressure during isovolumetric inspiratory efforts and ventilation against inspiratory loading. $J$ Appl Physiol (1985) 126, 699-707.

442 Bakdash JZ \& Marusich LR. (2017). Repeated Measures Correlation. Front Psychol 8, 456.

444 Baydur A, Behrakis PK, Zin WA, Jaeger M \& Milic-Emili J. (1982). A simple method for assessing the validity of the esophageal balloon technique. Am Rev Respir Dis 126, 788-791. 
Deffieux T, Gennisson JL, Tanter M \& Fink M. (2008). Assessment of the mechanical properties of the musculoskeletal system using 2-D and 3-D very high frame rate ultrasound. IEEE Trans Ultrason Ferroelectr Freq Control 55, 2177-2190.

Dres M \& Demoule A. (2020). Monitoring diaphragm function in the ICU. Curr Opin Crit Care 26, 18-25.

Geary CM, Welch JF, McDonald MR, Peters CM, Leahy MG, Reinhard PA \& Sheel AW. (2019). Diaphragm fatigue and inspiratory muscle metaboreflex in men and women matched for absolute diaphragmatic work during pressure-threshold loading. $J$ Physiol 597, 4797-4808.

Goligher EC, Laghi F, Detsky ME, Farias P, Murray A, Brace D, Brochard LJ, Bolz SS, Rubenfeld GD, Kavanagh BP \& Ferguson ND. (2015). Measuring diaphragm thickness with ultrasound in mechanically ventilated patients: feasibility, reproducibility and validity. Intensive Care Med 41, 642-649.

464

465 Gronlund C, Claesson K \& Holtermann A. (2013). Imaging two-dimensional mechanical 466 waves of skeletal muscle contraction. Ultrasound Med Biol 39, 360-369. $620-624$. 2005. 
Kabitz HJ, Walker D, Walterspacher S \& Windisch W. (2007). Controlled twitch mouth pressure reliably predicts twitch esophageal pressure. Respir Physiol Neurobiol 156, 276-282.

478

Laghi F, Harrison MJ \& Tobin MJ. (1996). Comparison of magnetic and electrical phrenic nerve stimulation in assessment of diaphragmatic contractility. J Appl Physiol (1985) 80, 1731-1742.

Laveneziana P, Albuquerque A, Aliverti A, Babb T, Barreiro E, Dres M, Dubé B-P, Fauroux B, Gea J, Guenette JA, Hudson AL, Kabitz H-J, Laghi F, Langer D, Luo Y-M, Alberto Neder J, O'Donnell D, Polkey MI, Rabinovich RA, Rossi A, Series F, Similowski T, Spengler C, Vogiatzis I \& Verges S. (2019). ERS Statement on Respiratory Muscle Testing at Rest and during Exercise. European Respiratory Journal, 1801214.

Loupas T, Powers JT \& Gill RW. (1995). An axial velocity estimator for ultrasound blood flow imaging, based on a full evaluation of the Doppler equation by means of a twodimensional autocorrelation approach. IEEE Transactions on Ultrasonics, Ferroelectrics, and Frequency Control 42, 672-688.

Man WD, Moxham J \& Polkey MI. (2004). Magnetic stimulation for the measurement of respiratory and skeletal muscle function. Eur Respir J 24, 846-860.

Mojoli F, Chiumello D, Pozzi M, Algieri I, Bianzina S, Luoni S, Volta CA, Braschi A \& Brochard L. (2015). Esophageal pressure measurements under different conditions of intrathoracic pressure. An in vitro study of second generation balloon catheters. 
Montaldo G, Tanter M, Bercoff J, Benech N \& Fink M. (2009). Coherent plane-wave compounding for very high frame rate ultrasonography and transient elastography. IEEE Trans Ultrason Ferroelectr Freq Control 56, 489-506.

Oppersma E, Hatam N, Doorduin J, van der Hoeven JG, Marx G, Goetzenich A, Fritsch S, Heunks LMA \& Bruells CS. (2017). Functional assessment of the diaphragm by speckle tracking ultrasound during inspiratory loading. J Appl Physiol (1985) 123, 1063-1070.

R Core Team. (2020). R: A Language and Environment for Statistical Computing. R Foundation for Statistical Computing, Vienna, Austria.

Rossi S, Hallett M, Rossini PM \& Pascual-Leone A. (2011). Screening questionnaire before TMS: an update. Clin Neurophysiol 122, 1686.

Sandrin L, Catheline S, Tanter M, Hennequin X \& Fink M. (1999). Time-resolved pulsed elastography with ultrafast ultrasonic imaging. Ultrason Imaging 21, 259-272.

Shahgholi L, Baria MR, Sorenson EJ, Harper CJ, Watson JC, Strommen JA \& Boon AJ. (2014). Diaphragm depth in normal subjects. Muscle Nerve 49, 666-668.

Similowski T, Fleury B, Launois S, Cathala HP, Bouche P \& Derenne JP. (1989). Cervical magnetic stimulation: a new painless method for bilateral phrenic nerve stimulation in conscious humans. J Appl Physiol (1985) 67, 1311-1318. 
Similowski T, Gauthier AP, Yan S, Macklem PT \& Bellemare F. (1993). Assessment of diaphragm function using mouth pressure twitches in chronic obstructive pulmonary disease patients. Am Rev Respir Dis 147, 850-856.

Spiesshoefer J, Henke C, Herkenrath S, Brix T, Randerath W, Young P \& Boentert M. (2019). Transdiapragmatic pressure and contractile properties of the diaphragm following magnetic stimulation. Respir Physiol Neurobiol 266, 47-53.

Teixeira A, Demoule A, Verin E, Morelot-Panzini C, Series F, Straus C \& Similowski T. (2007). Superiority of nasal mask pressure over mouth pressure, as a surrogate of diaphragm twitch-related esophageal pressure, in healthy humans. Respir Physiol Neurobiol 159, 236-240.

Tuinman PR, Jonkman AH, Dres M, Shi ZH, Goligher EC, Goffi A, de Korte C, Demoule A \& Heunks L. (2020). Respiratory muscle ultrasonography: methodology, basic and advanced principles and clinical applications in ICU and ED patients-a narrative review. Intensive Care Med 46, 594-605.

Ueki J, De Bruin PF \& Pride NB. (1995). In vivo assessment of diaphragm contraction by ultrasound in normal subjects. Thorax 50, 1157-1161.

\section{Umbrello M, Formenti P, Longhi D, Galimberti A, Piva I, Pezzi A, Mistraletti G, Marini JJ} \& Iapichino G. (2015). Diaphragm ultrasound as indicator of respiratory effort in critically ill patients undergoing assisted mechanical ventilation: a pilot clinical study. Crit Care 19, 161. 
553 Vivier E, Mekontso Dessap A, Dimassi S, Vargas F, Lyazidi A, Thille AW \& Brochard L. (2012). Diaphragm ultrasonography to estimate the work of breathing during noninvasive ventilation. Intensive Care Med 38, 796-803.

556

557 Welch JF, Archiza B, Guenette JA, West CR \& Sheel AW. (2018). Sex differences in 558 diaphragmatic fatigue: the cardiovascular response to inspiratory resistance. J Physiol 596, 4017-4032.

561 Windisch W, Kabitz HJ \& Sorichter S. (2005). Influence of different trigger techniques on 562 twitch mouth pressure during bilateral anterior magnetic phrenic nerve stimulation. (1994). Comparison of cervical magnetic stimulation and bilateral percutaneous electrical stimulation of the phrenic nerves in normal subjects. Eur Respir J 7, 17881792.

569

Yan S, Gauthier AP, Similowski T, Macklem PT \& Bellemare F. (1992). Evaluation of human diaphragm contractility using mouth pressure twitches. Am Rev Respir Dis 145, 1064-1069. 


\section{Tables}

576 Table 1. Within day reliability of twitch transdiaphragmatic pressure (Pditw), maximal

577 diaphragm tissue velocity ( $\left.\mathrm{Vdi}_{\max }\right)$ and diaphragm thickening fraction (TFdi $\left.{ }_{\text {tw }}\right)$ for all

578 stimulations. SEM, standard error of measurement; ICC, intraclass correlation coefficient; $579 \quad[95 \% \mathrm{CI}], 95 \%$ confidence interval.

580

\begin{tabular}{lccc}
\hline Variable & Mean (SD) & $\begin{array}{c}\text { SEM } \\
{[\mathbf{9 5} \% \mathbf{C I}]}\end{array}$ & $\begin{array}{c}\text { ICC } \\
{[\mathbf{9 5} \% \mathbf{C I}]}\end{array}$ \\
\hline $\mathrm{Pdi}_{\mathrm{tw}}\left(\mathrm{cmH}_{2} \mathrm{O}\right)$ & $11.6(9.5)$ & $1.55[1.39 ; 1.75]$ & $0.97[0.96 ; 0.98]$ \\
$\mathrm{Vdi}_{\max }\left({\left.\mathrm{mm} . \mathrm{s}^{-1}\right)}^{5.6(5.0)}\right.$ & $1.89[1.70 ; 2.13]$ & $0.86[0.81 ; 0.90]$ \\
& & & \\
$\mathrm{TFdi}_{\mathrm{tw}}(\%)$ & $18.7(15.6)$ & $10.41[9.38 ; 11.76]$ & $0.56[0.43 ; 0.66]$ \\
\hline
\end{tabular}

581

582 


\section{Figures}

584 Figure 1. A. Typical B-Mode image of the diaphragm using conventional ultrasound 585 imaging. Conventional ultrasound uses focused pulses, allowing high image quality but 586 relatively low sampling rate (a few tens per second). The diaphragm can be identified as a 587 three-layers structure superficial to the liver. The echogenic pleura and peritoneum layers 588 surround the muscular layer of the diaphragm. B. The diaphragm is imaged using the custom 589 ultrafast ultrasound sequence used in this study. Noteworthy, ultrafast ultrasound allows very 590 high frame rate but limited contrast of anatomic structures in comparison to standard US 591 imaging.

592 Figure 2. Experimental setup and procedure for recording pressure and ultrafast ultrasound

593 images. The participants were asked to bend their neck forward and the central hole of the 594 coil was positioned on the spinous process of the seventh cervical vertebra. Recording of 595 pressure signals was initiated $1000 \mathrm{~ms}$ before the onset of ultrasound recording. Cervical 596 magnetic stimulation was applied $100 \mathrm{~ms}$ after the onset of a 500-ms ultrafast ultrasound 597 acquisition.

598 Figure 3. Diaphragm tissue velocity (Vdi) over time along the longitudinal axis of the 599 ultrasound probe. Cervical magnetic stimulation occurs at $100 \mathrm{~ms}$ and is indicated by the 600 grey ribbon.

601 Figure 4. Typical ultrasound and physiological recordings at 40 (left), 70 (center) and 100 $602 \%$ (right) of stimulator intensity. The central pixel of each B-Mode image was used to 603 generate the M-Mode images (upper panel). Pleura (dashed) and peritoneum (solid) layers 604 displacement are presented in the second panel. Diaphragm tissue velocity (Vdi) is presented 605 in the third panel. Lastly, the transdiaphragmatic (Pdi), esophageal (Pes) and gastric (Pga) 606 pressures are displayed in the bottom panel. The dotted vertical lines at $100 \mathrm{~ms}$ indicate the 607 onset of cervical magnetic stimulation.

608 Figure 5. Esophageal (Pestw, A.) and gastric (Pgatw, B.) twitch pressures at different 609 stimulation intensities. Box plots present first and third quartiles, in addition to the median. 610 The range over which the data spread out is defined by the whiskers. 
611 Figure 6. Twitch transdiaphragmatic pressure (Pditw, A.), maximal diaphragm tissue velocity 612 (Vdimax, B.), and diaphragm thickening fraction (TFditw, C.) according to stimulation 613 intensities. Box plots present first and third quartiles, in addition to the median. The range 614 over which the data spread out is defined by the whiskers. Repeated measure ANOVAs were 615 used to assess the effect of stimulation intensity on Pditw, Vdimax, and TFditw. Tukey’s post616 hoc tests were conducted if a significant main effect of intensity was found. *, significant 617 difference with the preceding stimulation intensity (i.e. -10\%); \#, significant difference with 618 the second preceding stimulation intensity (i.e. $-20 \%$ ). Averaged data points for each 619 participant are displayed for Pditw (D.), Vdimax (E.), and TFditw (F.). Red points on panels D, $620 \mathrm{E}$, and $\mathrm{F}$ indicate supramaximality for the given parameter.

621 Figure 7. Averaged data points for each participant regarding the relationships between 622 twitch transdiaphragmatic pressure (Pditw) and maximal diaphragm tissue velocity (Vdimax, 623 A.) and between Pditw and diaphragm thickening fraction (TFditw, B.). 

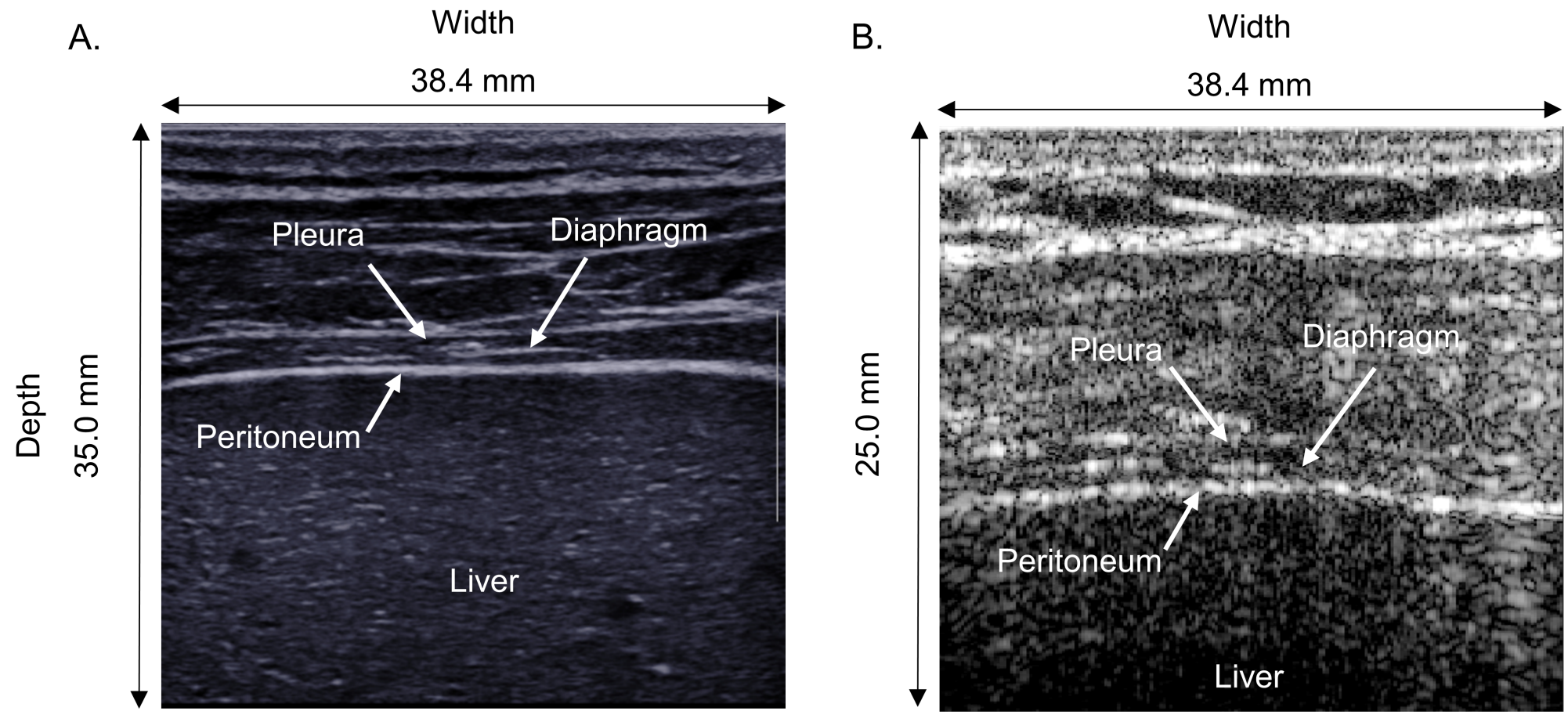


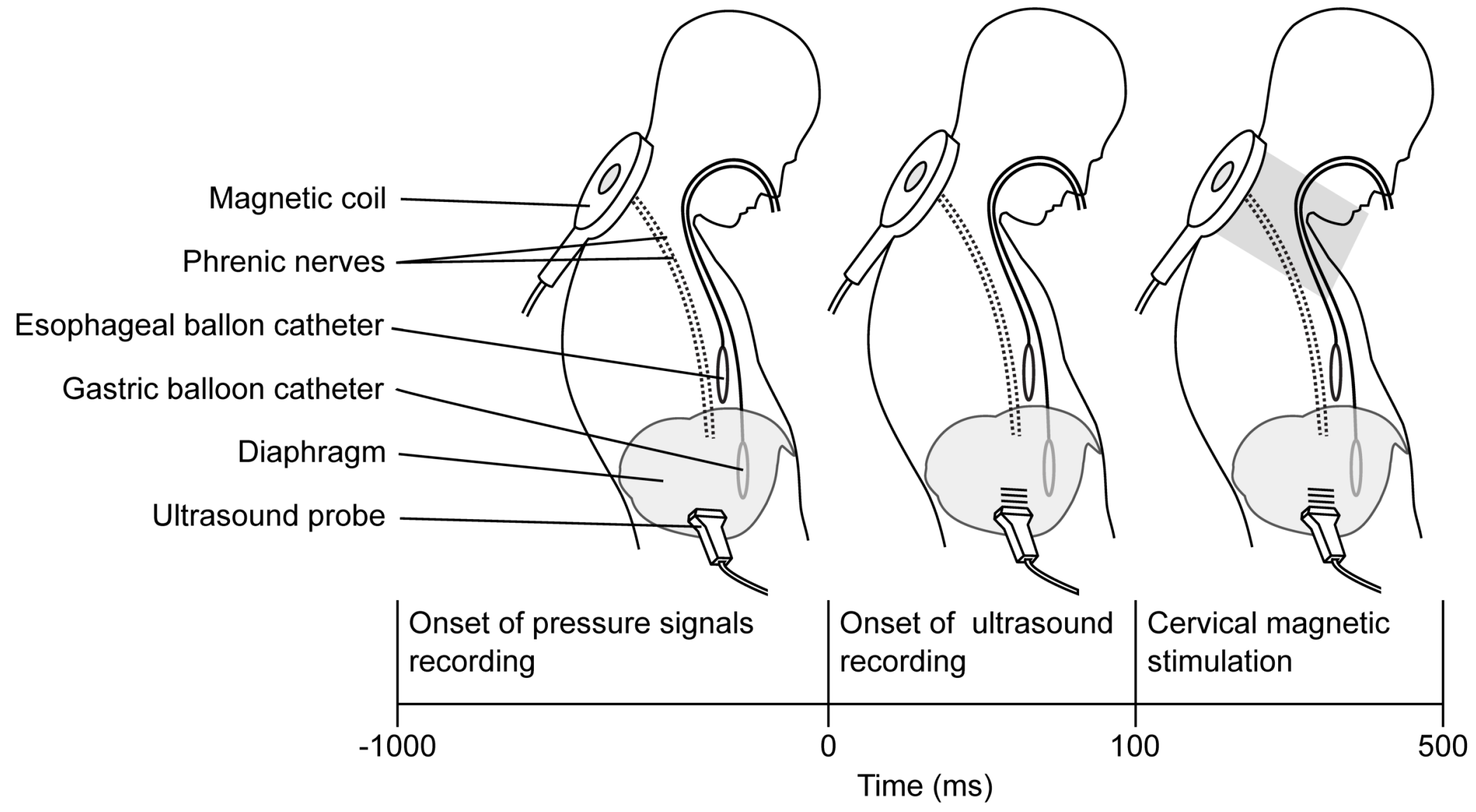




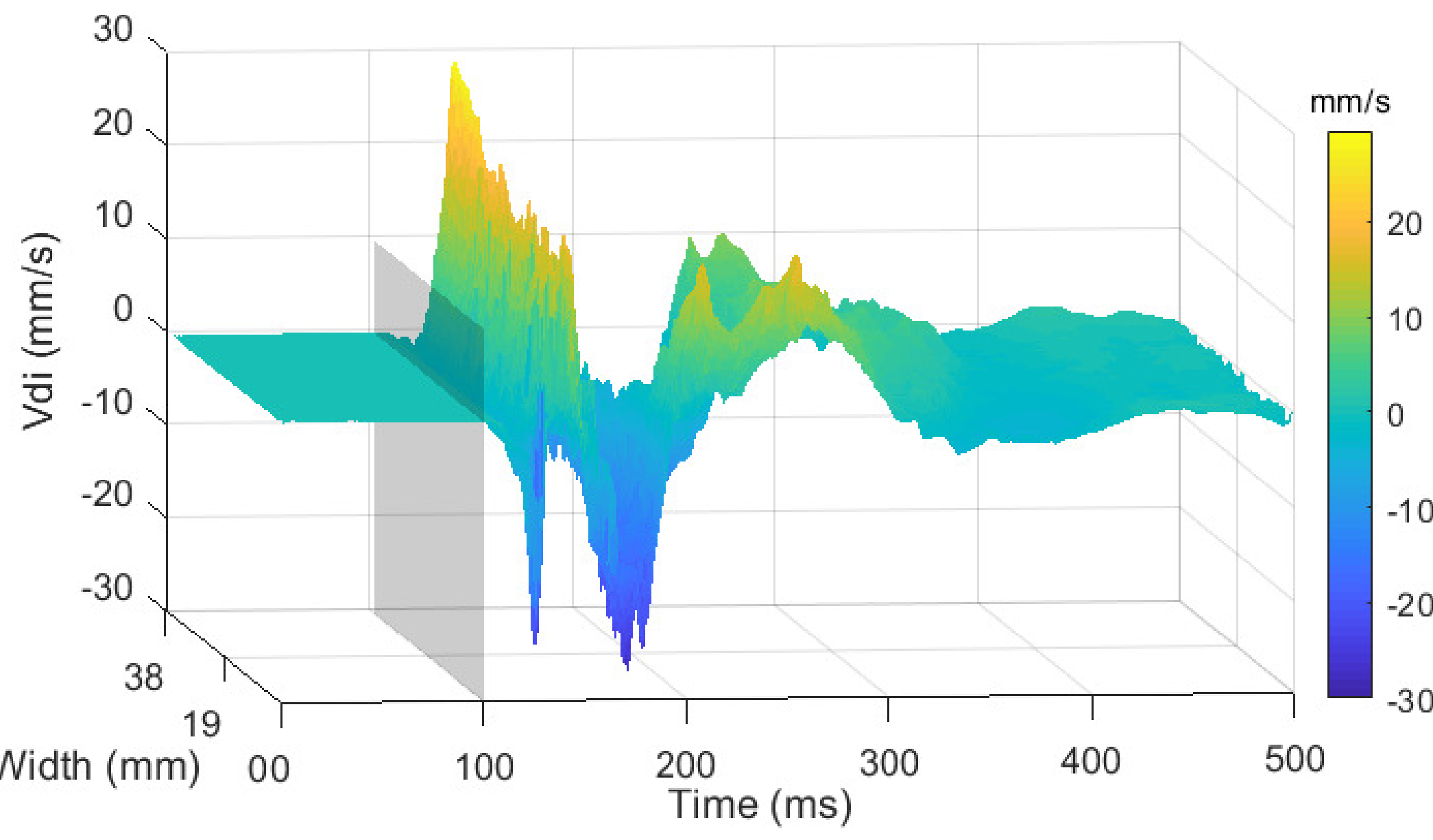




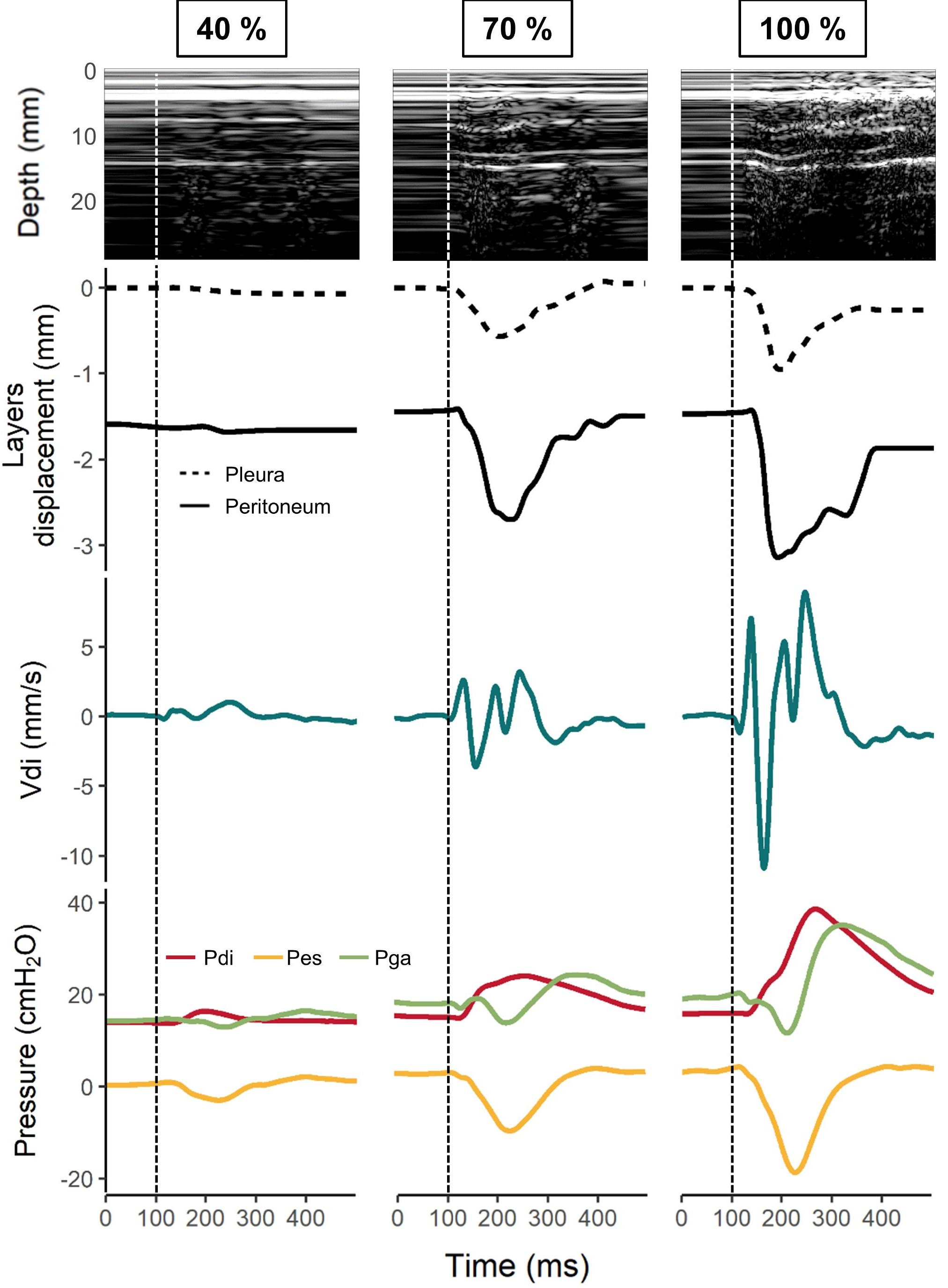


A.

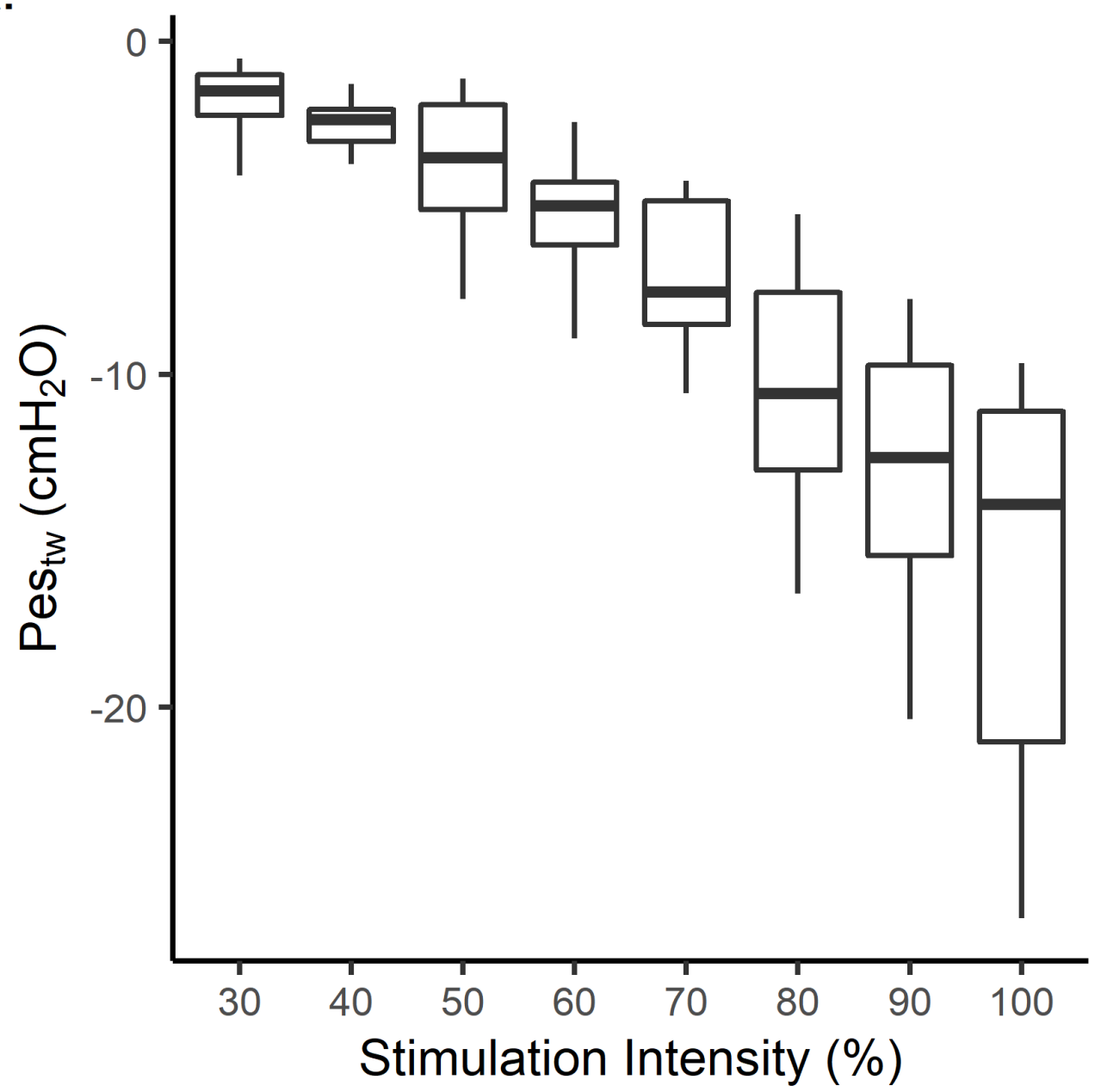

B.

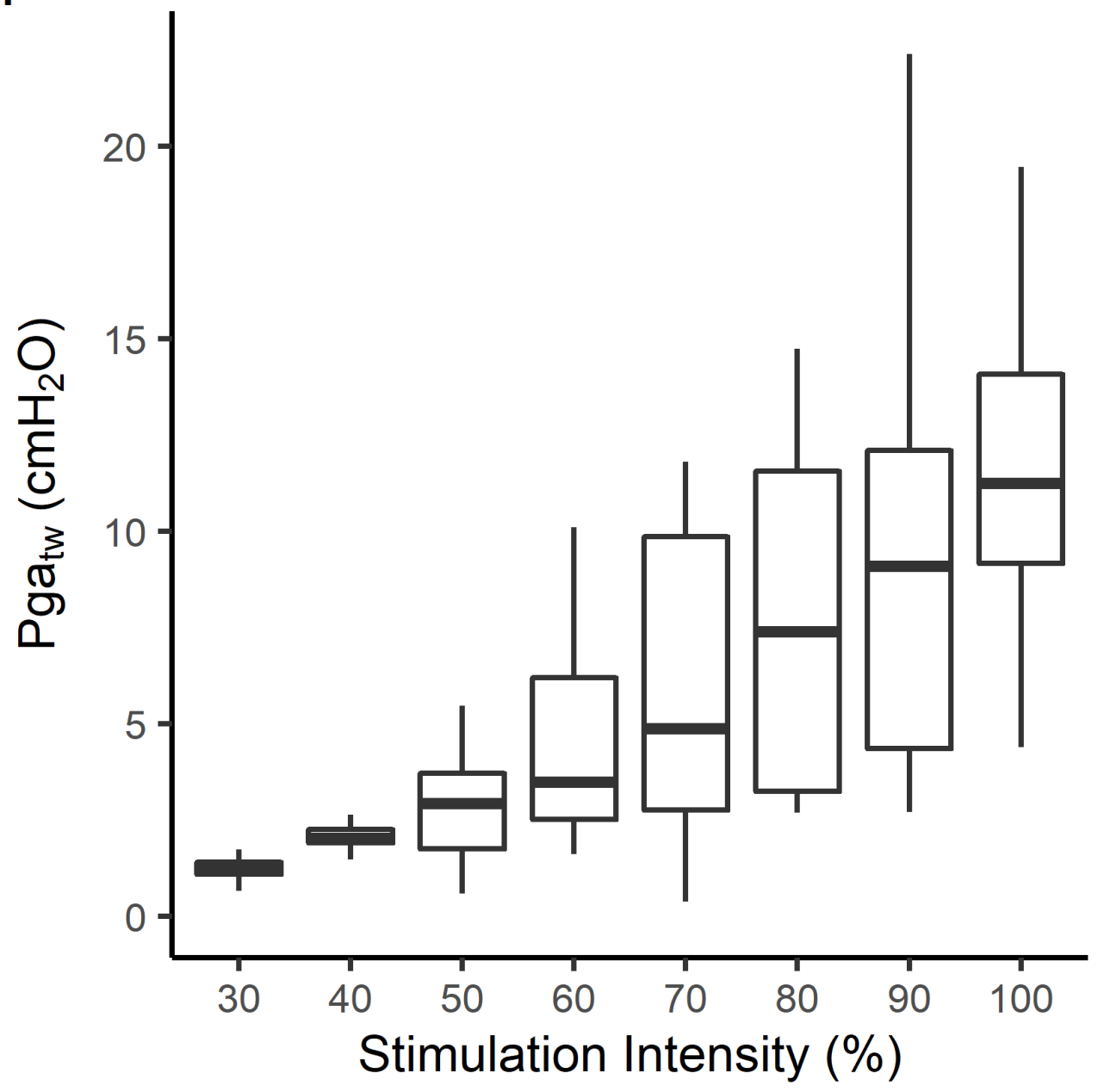


A.

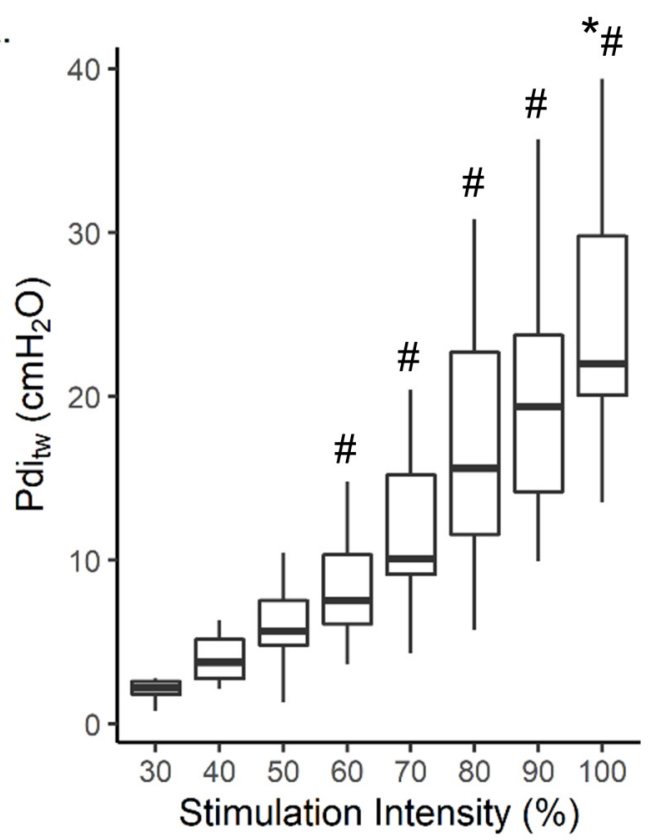

D.

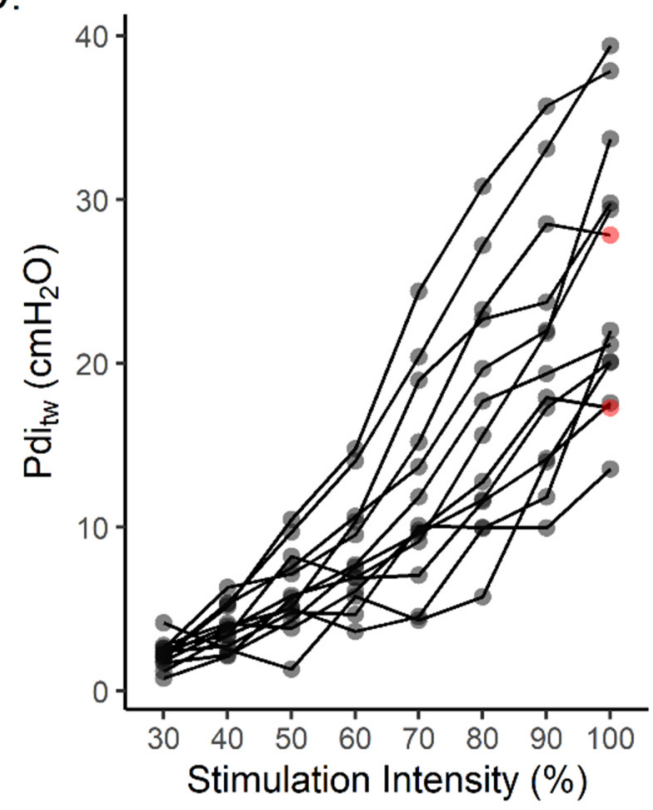

B.

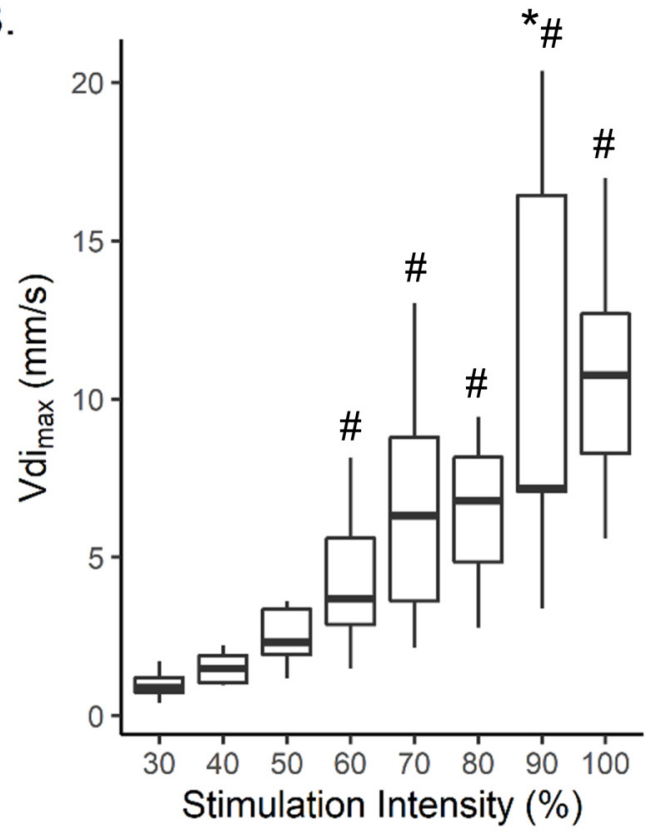

E.

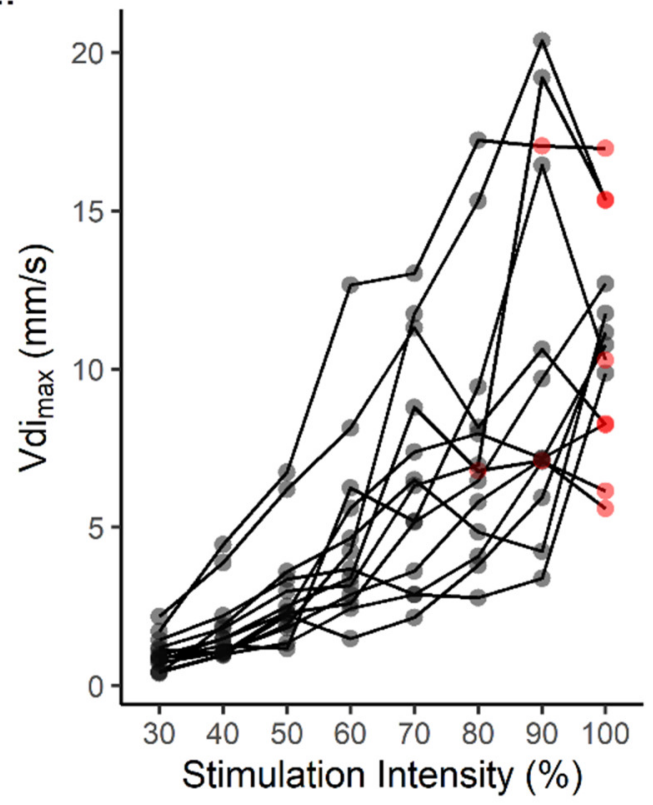

C.

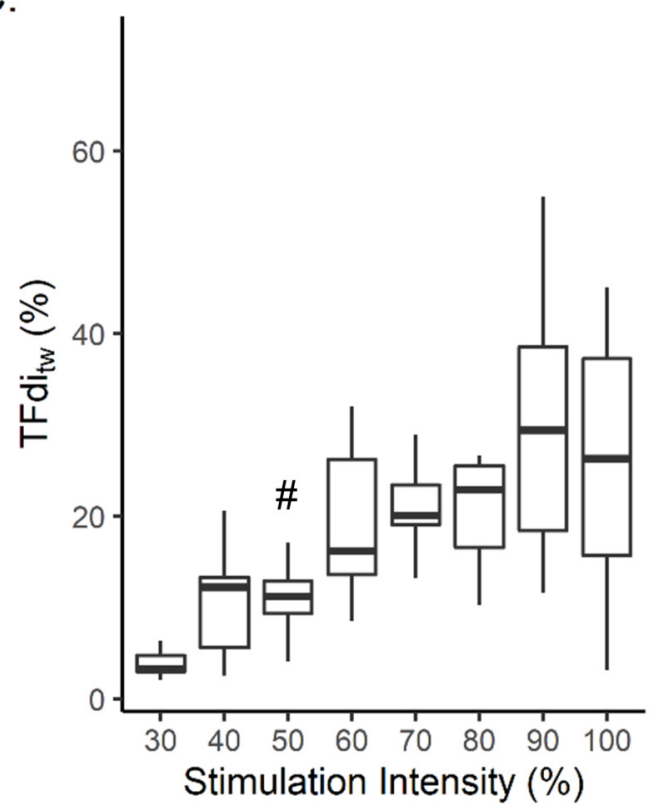

F.

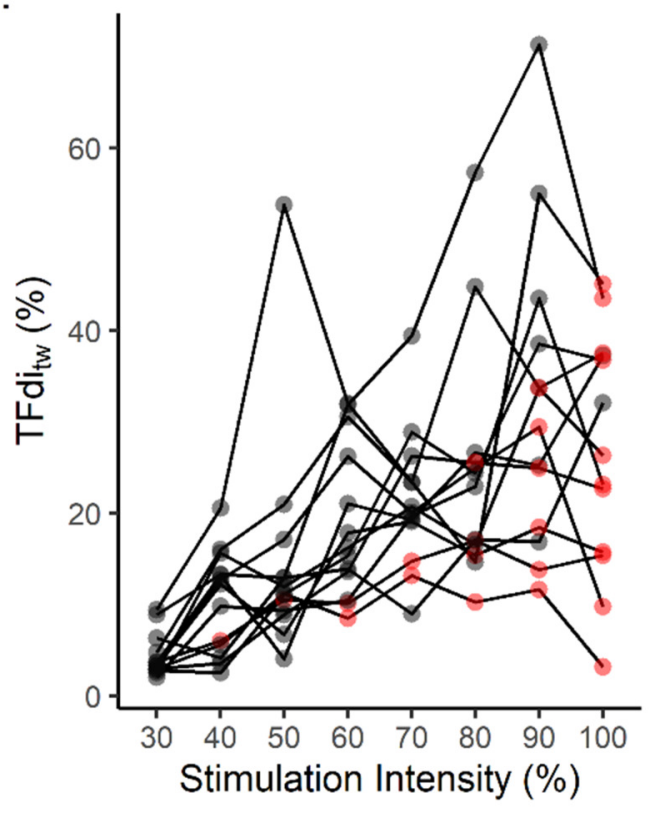


A.

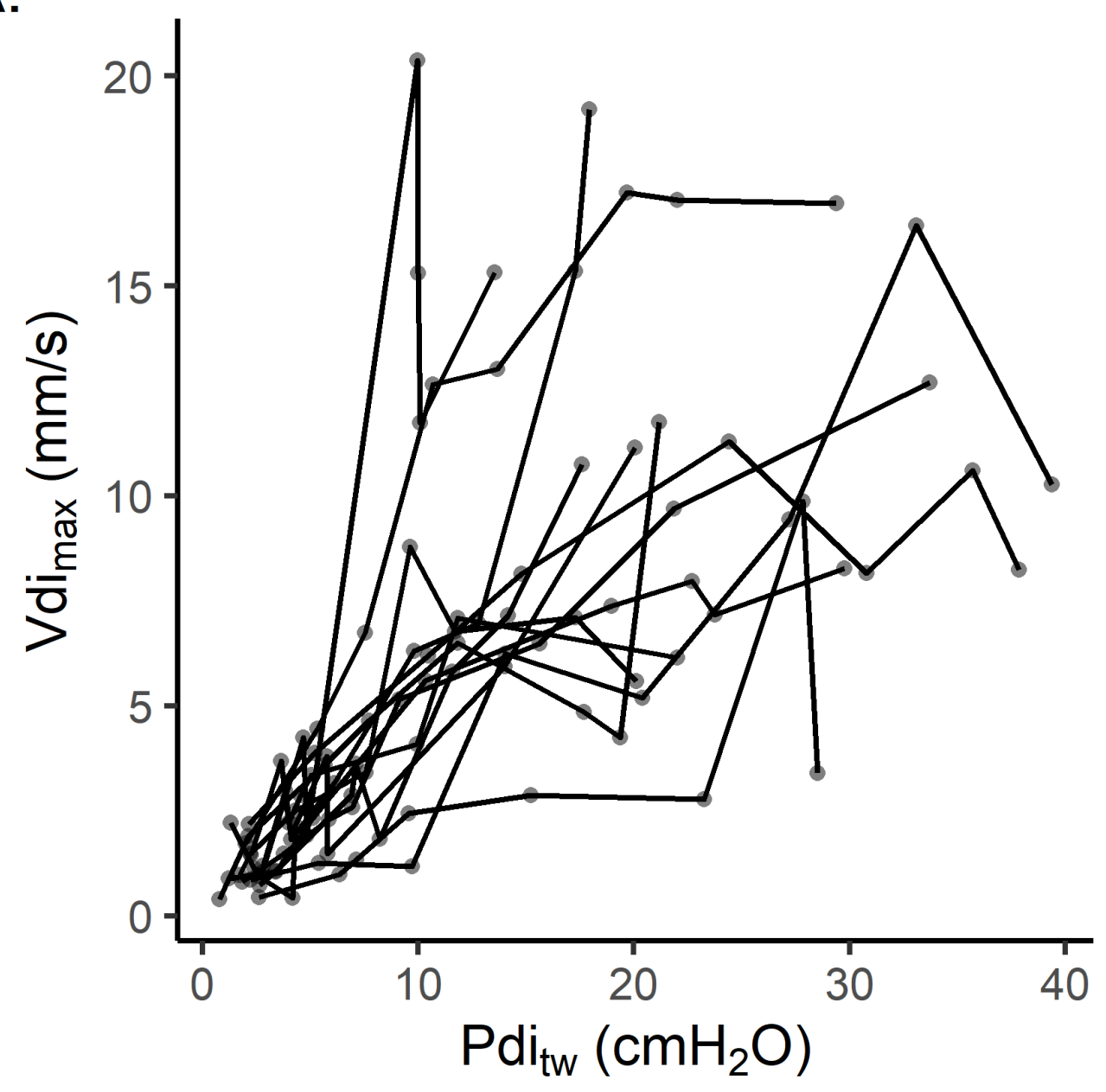

B.

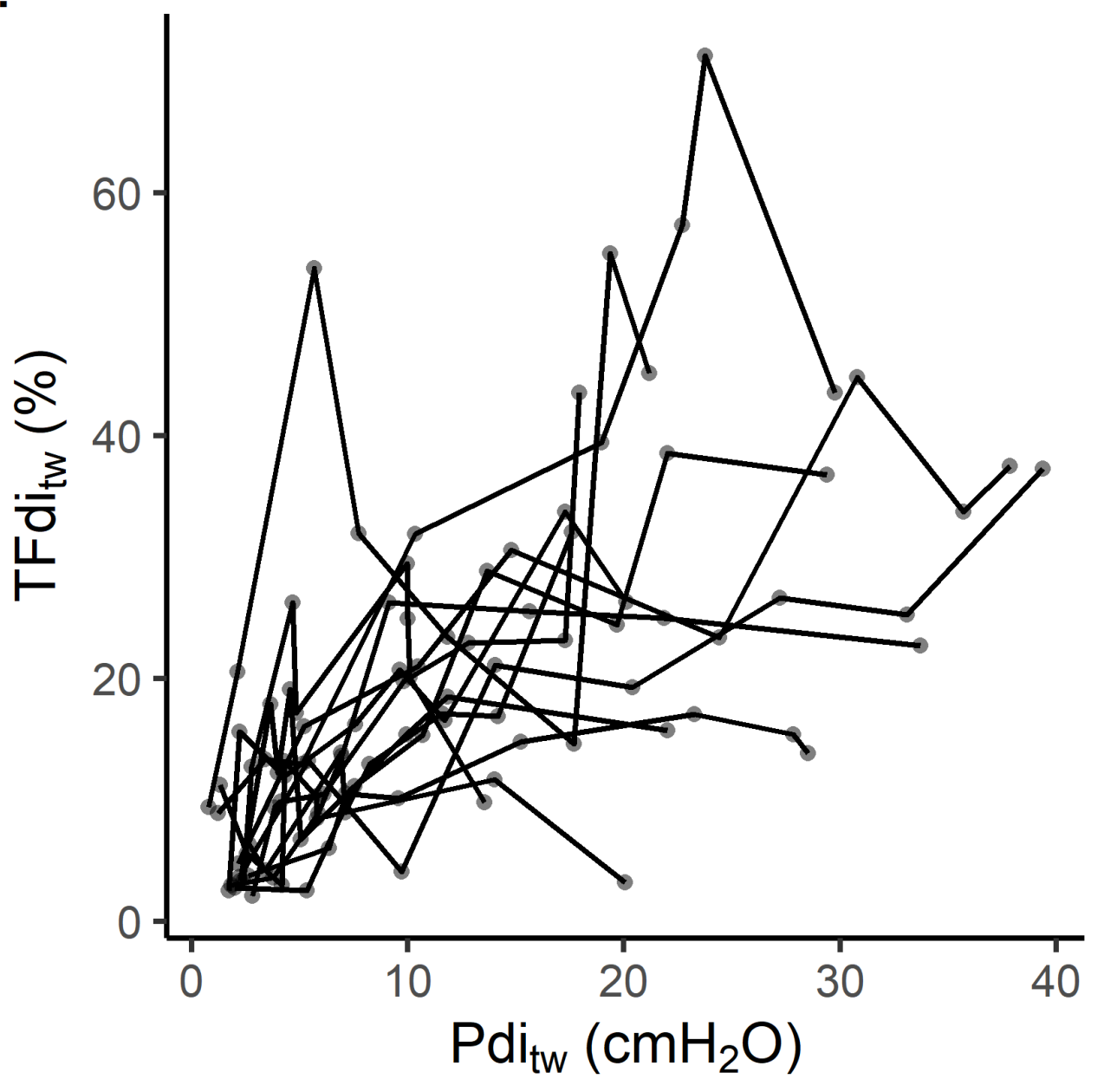

